\title{
Modeling the Effect of Propofol and Remifentanil Combinations for Sedation-Analgesia in Endoscopic Procedures Using an Adaptive Neuro Fuzzy Inference System (ANFIS)
}

\author{
P. L. Gambús, MD,* E. W. Jensen, MSc, PhD, † M. Jospin, MSc, $†$ X. Borrat, MD,* G. Martínez Pallí, MD,* \\ J. Fernández-Candil, MD,* J. F. Valencia, MSc, $\uparrow$ X. Barba, CRNA,* P. Caminal, MSc, PhD, $\uparrow$ \\ and I. F. Trocóniz, PhD
}

BACKGROUND: The increasing demand for anesthetic procedures in the gastrointestinal endoscopy area has not been followed by a similar increase in the methods to provide and control sedation and analgesia for these patients. In this study, we evaluated different combinations of propofol and remifentanil, administered through a target-controlled infusion system, to estimate the optimal concentrations as well as the best way to control the sedative effects induced by the combinations of drugs in patients undergoing ultrasonographic endoscopy.

METHODS: One hundred twenty patients undergoing ultrasonographic endoscopy were randomized to receive, by means of a target-controlled infusion system, a fixed effect-site concentration of either propofol or remifentanil of 8 different possible concentrations, allowing adjustment of the concentrations of the other drug. Predicted effect-site propofol $\left(\mathrm{C}_{\mathrm{e}}\right.$ pro) and remifentanil $\left(\mathrm{C}_{\mathrm{e}}\right.$ remi) concentrations, parameters derived from auditory evoked potential, autoregressive auditory evoked potential index (AAl/2) and electroencephalogram (bispectral index [BIS] and index of consciousness [loC]) signals, as well as categorical scores of sedation (Ramsay Sedation Scale [RSS] score) in the presence or absence of nociceptive stimulation, were collected, recorded, and analyzed using an Adaptive Neuro Fuzzy Inference System. The models described for the relationship between $\mathrm{C}_{\mathrm{e}}$ pro and $\mathrm{C}_{\mathrm{e}}$ remi versus $\mathrm{AAI} / 2$, BIS, and loC were diagnosed for inaccuracy using median absolute performance error (MDAPE) and median root mean squared error (MDRMSE), and for bias using median performance error (MDPE). The models were validated in a prospective group of 68 new patients receiving different combinations of propofol and remifentanil. The predictive ability $\left(P_{k}\right)$ of $\mathrm{AAI} / 2$, BIS, and loC with respect to the sedation level, RSS score, was also explored.

RESULTS: Data from 110 patients were analyzed in the training group. The resulting estimated models had an MDAPE of 32.87, 12.89, and 8.77; an MDRMSE of 17.01, 12.81, and 9.40; and an MDPE of $-1.86,3.97$, and 2.21 for $\mathrm{AAI} / 2$, BIS, and IoC, respectively, in the absence of stimulation and similar values under stimulation. $\mathrm{P}_{\mathrm{k}}$ values were $0.82,0.81$, and $0.85 \mathrm{for} \mathrm{AAl} / 2$, $\mathrm{BIS}$, and IoC, respectively. The model predicted the prospective validation data with an MDAPE of 34.81, 14.78, and 10.25; an MDRMSE of 16.81, 15.91, and 11.81; an MDPE of -8.37 , 5.65, and -1.43; and $\mathrm{P}_{\mathrm{k}}$ values of $0.81,0.8$, and 0.8 for $\mathrm{AAI} / 2$, BIS, and IoC, respectively. CONCLUSION: A model relating $\mathrm{C}_{\mathrm{e}}$ pro and $\mathrm{C}_{\mathrm{e}}$ remi to $\mathrm{AAI} / 2$, $\mathrm{BIS}$, and IoC has been developed and prospectively validated. Based on these models, the $\left(\mathrm{C}_{\mathrm{e}}\right.$ pro, $\mathrm{C}_{\mathrm{e}}$ remi) concentration pairs that provide an RSS score of 4 range from $\left(1.8 \mu \mathrm{g} \cdot \mathrm{mL}^{-1}, 1.5 \mathrm{ng} \cdot \mathrm{mL}^{-1}\right)$ to $\left(2.7 \mu \mathrm{g} \cdot \mathrm{mL}^{-1}, 0 \mathrm{ng} \cdot \mathrm{mL}^{-1}\right)$. These concentrations are associated with AAI/2 values of 25 to 30 , BIS of 71 to 75 , and loC of 72 to 76. The presence of noxious stimulation increases the requirements of $C_{e}$ pro and $C_{e}$ remi to achieve the same degree of sedative effects. (Anesth Analg 2011;112:331-9)

$\mathrm{T}$ he requirements for administration of sedation and/or analgesia for minimally invasive diagnostic and therapeutic procedures have increased enormously. Providing anesthesia for gastrointestinal endos-

From the *Anesthesiology Department, Hospital Clinic de Barcelona, Barcelona; +Center for Research in Biomedical Engineering, Polytechnic University of Catalunya, Barcelona; and tDepartment of Pharmacy and Pharmaceutical Technology, School of Pharmacy, Universidad de Navarra, Pamplona, Spain. Accepted for publication August 31, 2010.

Supported by FIS (Fondo de Investigaciones Sanitarias, Instituto de Salud Carlos III, Health Department, Government of Spain) grant PI050072.

Presented in part at the ASA Annual Meeting, Chicago, IL, October 13-18, 2006. copy is a good example of this growing area of anesthetic activity. Sedation and analgesia increase patient comfort and provide better working conditions for endoscopists.

Several drugs or drug combinations are currently used in sedation-analgesia. Propofol is the most frequently used,

Address correspondence and reprint requests to P.L. Gambús, MD, PharmacoAnesthetics Research group (PARG), Servicio de Anestesiología, Hospital Clinic de Barcelona, Villarroel 170, 08036 Barcelona, Spain. Address e-mail to plgambus@hospitalclinic.org.

Copyright (C) 2011 International Anesthesia Research Society DOI: 10.1213/ANE.0b013e3182025a70 
combined or not with remifentanil. Target-controlled infusions (TCIs) allow the administration of IV drugs to achieve and maintain a certain target concentration in either plasma or biophase according to pharmacokinetic-pharmacodynamic (PK-PD) models. ${ }^{1}$

Although the demand for sedation-analgesia has increased because of patient and endoscopist requirements, there has not been a proportional increase in establishing the adequate drug combination or the optimal way to control sedation. Different systems have been used to assess the level of patient hypnosis. Besides the observer evaluation of sedation by means of categorical scores such as the Ramsay Sedation Scale (RSS) score ${ }^{2}$ or the Observer's Assessment of Alertness/Sedation (OAA/S) score, ${ }^{3}$ methods based on the analysis of the spontaneous electroencephalographic (EEG) signal such as the bispectral index (BIS), ${ }^{4}$ or based on the analysis of the auditory evoked potentials (AEPs) such as the autoregressive auditory evoked potential index (AAI/2), ${ }^{5}$ have been used for surgical procedures. Most of the studies on sedation recommend target BIS values in the range of 80 to 65 depending on the depth of sedation (RSS score 3-4 or OAA/S score 3-2). Because patients undergoing endoscopic explorations are for the most part outpatients, drug effects must be carefully titrated to warrant a fast hospital discharge after the procedure is finished. Methods to optimize the control of sedative and analgesic effects would improve adjusting drug administration to achieve exactly the level of effect desired.

Adaptive Neurofuzzy Inference System (ANFIS) is a fuzzy logic-based modeling approach that establishes the relationship between variables based on a nonrestricted mathematical structure, but simply going through the data with no prior assumption of the mathematical relationship between variables. ${ }^{6,7}$ The advantage of using ANFIS is that it is a data-driven approach that does not assume an underlying mathematical model governing the relationship between the anesthetic drugs and the response-effect.

The hypothesis of this project is that available methods to control hypnosis during surgery could be used to control the sedative effects induced by different combinations of propofol and remifentanil, administered by means of a TCI system, in patients undergoing ultrasonographic endoscopy (USE). Based on the above, the objectives were to use the model predictions to define the optimal combination of propofol and remifentanil to keep patients unconscious, although arousable by tactile stimulation, and also to define the recommended ranges of $\mathrm{BIS}, \mathrm{AAI} / 2$, and the newly introduced index of consciousness (IoC) monitor associated with that level of sedation.

\section{METHODS}

This was a randomized, observational, nonblinded study. After receiving approval from the Ethics Committee of Hospital Clinic de Barcelona and signed informed consent, 120 patients scheduled to undergo USE under sedationanalgesia were randomized to 1 of 8 different groups according to the drug combination received. USE was chosen because it is a relatively long procedure, approximately 1 hour, with periods of stability of effect and others when the intensity of endoscopic stimulus is evident,

\begin{tabular}{|c|c|c|}
\hline $\begin{array}{l}\text { Target levels of } \\
\text { the drugs }\end{array}$ & $\begin{array}{l}\text { Range of } C_{e} \text { of the } \\
\text { other drug }\end{array}$ & $\begin{array}{c}\text { No. of } \\
\text { individuals }\end{array}$ \\
\hline \multicolumn{3}{|l|}{ Propofol $\left(\mu \mathrm{g} \cdot \mathrm{mL}^{-1}\right)$} \\
\hline 0 & 1.5-3.5 & 10 \\
\hline 1.5 & $0.5-3.8$ & 16 \\
\hline 2 & $0.6-2.7$ & 15 \\
\hline 3 & $0.5-2$ & 12 \\
\hline \multicolumn{3}{|c|}{ Remifentanil $\left(\mathrm{ng} \cdot \mathrm{mL}^{-1}\right)$} \\
\hline 0 & $1.4-3.8$ & 14 \\
\hline 0.5 & $1.5-4$ & 16 \\
\hline 1 & $0.5-3.8$ & 14 \\
\hline 2 & $1-2.8$ & 13 \\
\hline
\end{tabular}

Patients assigned propofol 0 required remifentanil concentrations ranging from 1.5 to $3.5 \mathrm{ng} \cdot \mathrm{mL}^{-1}$. Similar interpretation for the remaining 7 groups.

allowing study of the repercussion of painful stimulus on the level of sedation.

A second group of 68 patients undergoing the same endoscopic procedure was also included in the study and used to prospectively validate the concentration-effect models. In this group, propofol and remifentanil were not randomized but administered according to the clinical judgment of the attending anesthesiologist.

\section{Drug Administration}

Using a crisscross design for drug administration, patients received a combination of propofol and remifentanil in which 1 of the drugs was fixed depending on the group that the patient was assigned to by the randomization process. The details of the target concentrations defining the 8 groups are shown in Table 1. The fixed drug was kept constant throughout the whole procedure and the other drug, nonfixed, was allowed to vary according to clinical demands. Crisscross design warrants the collection of enough data at all possible drug combinations of propofol and remifentanil. ${ }^{8}$

Propofol and remifentanil were infused using a TCI system (FreseniusVial; Chemin de Fer, Béziers, France). The TCI system administered propofol according to the predictions of the PK-PD model described by Schnider et al.9,10 and remifentanil according to the predictions of the PK-PD model described by Minto et al. ${ }^{11}$ In both cases, the TCI was targeting the effect site.

\section{Effect Measurements}

After arrival in the USE room, every patient was routinely monitored, including electrocardiogram, noninvasive arterial blood pressure $(\mathrm{ABP})$, pulse oximetry $\left(\mathrm{Spo}_{2}\right)$, and respiratory rate using transcutaneous bioimpedance collected from the electrocardiogram electrodes.

BIS of the EEG was continuously measured using an A2000 monitor (software version 3.31) (Aspect Medical Systems, Newton, MA). A 4-electrode sensor was placed on the foreheads of the patients according to the manufacturer's instructions. The AAI/2 derived from AEP signal was recorded using the AEP monitor/2 (Danmeter, Odense, Denmark). A 3-electrode montage was used: middle forehead $(+)$, malar bone $(-)$, and left forehead electrode used as reference. From the same AAI/2 electrodes, the raw EEG signal was recorded and stored into a flash memory card. 
The IoC (Aircraft Medical, formerly Morpheus Medical, Barcelona, Spain) was calculated off-line from the raw EEG signal as a composite index combining spectral analysis and symbolic dynamics as described elsewhere. ${ }^{12,13}$

The RSS was used as measure of sedation. ${ }^{2}$ An optimal level of sedation, based on patients' preferences and endoscopists' demands was defined when the patient was unconscious, eyes closed, but arousable by tactile stimulation, which corresponds to an RSS score of 4 , a moderate degree of sedation. The RSS score was evaluated at random times during the procedure to avoid those factors correlated with time, which could confound the results of the RSS measurements.

The presence or absence of the tube inside the upper digestive tract of the patient was also recorded and considered as "stimulation" (STIM) when it was present and "no stimulation" (NO STIM) when absent.

\section{Data Collection}

Demographic patient data were collected. Data from the TCI system including infusion rates, target, plasma, and effect-site predicted concentrations of propofol ( $\mathrm{C}_{\mathrm{e}}$ pro $)$ and remifentanil $\left(\mathrm{C}_{\mathrm{e}}\right.$ remi), from the BIS monitor (BIS, electromyogram activity), from the A-Line monitor (AAI/2, raw EEG), and from the hemodynamics monitor (heart rate [HR], $\mathrm{ABP}, \mathrm{Spo}_{2}$, and respiratory rate) were also collected using the software Rugloop $\mathrm{II}^{\circledR}$ (Demed, Temse, Belgium). Data were automatically recorded with a resolution of 1 data point every 5 seconds and stored in the hard drive of the computer for posterior processing and analysis. MATLAB $^{\mathrm{TM}}$ (MathWorks, Inc., Natick, MA) was used to write the program that allows processing and synchronizing the data derived from the different monitors used to quantify drug effects.

\section{Clinical Management}

Patients were asked to lie in the left lateral decubitus position for at least 5 minutes before drug infusion started. In case of inadequate sedation after infusion started, hemodynamic depression ( $\mathrm{HR}<45 \mathrm{bpm}$ or mean ABP $<60 \mathrm{~mm}$ $\mathrm{Hg})$, respiratory depression $\left(\mathrm{Spo}_{2}<94 \%\right.$ or respiratory rate $<6$ breaths per minute), or any other event judged to endanger the patient or the normal conduct of the exploration by the attending anesthesiologist, clinical actions were undertaken, including, if necessary, intravascular volume infusion, atropine or ephedrine administration, variations in the fixed drug, or discontinuation of drug administration.

\section{Data Analysis}

ANFIS modeling was used to define the interaction of $\mathrm{C}_{\mathrm{e}}$ pro and $\mathrm{C}_{\mathrm{e}}$ remi with respect to the following effect measures: (1) AAI/2, (2) BIS, and (3) IoC, in the presence or absence of noxious stimulation, as defined previously. EEG and AEP data were used in the modeling process provided that signal quality was $>50 \%$.

ANFIS is a hybrid between a fuzzy logic system and a neural network. Its background and methodology have been described elsewhere ${ }^{7}$ and an application to the problem of evaluation of depth of anesthesia has also been published. ${ }^{14}$ In our case, the ANFIS system as implemented in MATLAB was fed with 2 inputs: $C_{e}$ pro and $C_{e}$ remi. Every input was "fuzzified" into 2 classes where the membership function was Gaussian. Each input presented to ANFIS is then assigned a class and a probability of belonging to that class. In our case, classes were "high propofol" versus "low propofol" and "high remifentanil" versus "low remifentanil," according to the predicted effect-site concentrations of each drug. The output function, which was of zero order, defuzzies the data back to crisp values. This means that any surface shape can result from the training, not necessarily a sigmoidal-shaped surface. The resulting surface will be the one that corresponds best to the relationship between input and output of the model, in terms of minimizing a least mean square function. In summary, each model had 2 inputs, $C_{e}$ pro and $C_{e}$ remi, and 1 output, either AAI/2, BIS, or IoC and the relationship is described by the surface that optimally describes it.

The number of training epochs chosen was 50 to reduce the training error. The model was fit to the data by iteratively minimizing the root mean square errors (RMSEs) using a backpropagation gradient descent method. ANFIS estimated the parameters of the models that best fit the data for the combinations of $\mathrm{C}_{\mathrm{e}}$ pro and $\mathrm{C}_{\mathrm{e}}$ remi with respect to the different measures of sedative effects studied.

\section{Evaluation of Model Performance}

To statistically evaluate the performance of the model, the percentage performance error (PE) defined by Varvel et al. ${ }^{15}$ as the difference between the observations and the predictions of the model divided by the predicted values was calculated for every data point recorded in each individual as follows:

$$
\mathrm{PE}=(\mathrm{Obs}-\text { Pred }) / \text { Pred }
$$

Where Obs means observations and Pred the corresponding value predicted by the model. The median of the PE (MDPE) and of the absolute values of PE (MDAPE) for every subject were computed for all patients included in the study. The median of all the individual MDPEs and MDAPEs was also calculated and reported as indicator of bias and inaccuracy, respectively. ${ }^{15}$

Because the range of the values taken by $\mathrm{AAI} / 2$, on one side, and by BIS and IoC, on the other side, are different for sedation, it might be worthwhile to consider an alternative measure of accuracy based on the difference between observations and predictions instead of normalizing it to the predicted values. For that purpose, the RMSE is also computed for each model. The RMSE is defined as:

$$
\text { RMSE }=\sqrt{\frac{1}{N} \sum(\text { Obs }- \text { Pred })^{2}}
$$

Where $N$ is the number of observation/prediction pairs. Similarly to MDPE and MDAPE, the value considered in this study is the median of the RMSE achieved for each patient (MDRMSE).

Different hypnotic effect measures were recorded during the study. To evaluate which was more accurate in predicting the level of patient sedation, the ability of the different effect measures used in predicting the degree of 
Table 2. Demographic Characteristics of the Patients in Both Groups

\begin{tabular}{lcc} 
& Training & Validation \\
Age $(\mathrm{y})$ & $61(19-83)$ & $62(22-83)$ \\
Weight $(\mathrm{kg})$ & $67(41-119)$ & $68(46-107)$ \\
Height $(\mathrm{cm})$ & $165.5(145-192)$ & $164.5(140-183)$ \\
Gender (male/female) & $72 / 38$ & $45 / 23$ \\
\hline
\end{tabular}

Data are median (range).

sedation, as measured by the RSS, was assessed by calculating the $\mathrm{P}_{\mathrm{k}}$ statistic as described by Smith et al., ${ }^{16}$ which has been extensively used elsewhere. ${ }^{17}$ At this step, $\mathrm{C}_{\mathrm{e}}$ pro and $\mathrm{C}_{\mathrm{e}}$ remi as well as hemodynamic measures ( $\left.\mathrm{ABP}, \mathrm{HR}\right)$ were also evaluated as predictors of the RSS values.

\section{Model Validation}

The model obtained based on the training group data was used to predict the values of AAI/2, BIS, and IoC induced by $\mathrm{C}_{\mathrm{e}}$ pro and $\mathrm{C}_{\mathrm{e}}$ remi in the prospective validation group. MDAPE, MDRMSE, and MDPE, as well as $P_{k}$ value, were also calculated for the studied measures of effect in the prospective validation group.

\section{Model Application}

To estimate the values of the hypnosis monitors associated with an RSS score of 4 , the values of $\mathrm{C}_{\mathrm{e}}$ pro and $\mathrm{C}_{\mathrm{e}}$ remi, captured from the TCI system, that induced an RSS score of 4 in the individuals of the training group were used as input to the different models to predict the values of $\mathrm{AAI} / 2$, BIS, and IoC associated with that level of sedation.

\section{RESULTS}

\section{Training Group}

Data from only 110 patients could be used in the data analysis process of the training group. Technical problems (accidental IV line disconnection, transient malfunction of the data collection system) prevented the collection of reliable data from the remaining 10 patients. Table 1 shows the number of individuals included in each group and the range of predicted concentrations of the drug adjusted to clinical demands for each fixed drug concentration. Demographic patient characteristics are shown in Table 2. The median (range) duration of each exploration was $71 \mathrm{~min}-$ utes (29-216 minutes).

A total of 1304 evaluations of RSS score were performed in the 110 patients from the training group. The median number of RSS score evaluations was 11 per patient. A total of 708 were performed without stimulation, whereas the remaining 596 were performed under stimulation.

With respect to neurophysiological measures, $>51,000$ data points were collected (approximately 23,000 without stimulation and 28,000 with stimulation).

In 90 training group patients, it was possible to maintain the randomized fixed concentration during the whole procedure. In the remaining 20, changes were made. The main reasons for these changes were an inadequate level of sedation, when the patient was too responsive, and when anesthesia was too deep for the level required to adequately conduct the procedure.

In the training group, there were 4 apnea events that required stopping drug infusion and manual ventilation support. In 3 patients, it was necessary to administer atropine for bradycardia (2 cases) or ephedrine for hypotension, respectively.

\section{Modeling}

The output function was of zero order, hence the total number of parameters in the model was 16. The results of the modeling process are presented in Figures 1 to 3 . Each graph shows the relationship between $\mathrm{C}_{\mathrm{e}} \mathrm{pro}, \mathrm{C}_{\mathrm{e}}$ remi and the different effects measured. The distribution of the observations in the 3-dimensional plot is summarized using cubes whose volumes are proportional to the number of observations encountered in each local region of this 3-dimensional space. For each graph, a fixed volume is assigned to the cube corresponding to the highest number of observations and the volume of all the remaining cubes is derived proportionally. This is intended to optimize the visualization for each plot, but attention should be given to the fact that 2 cubes of equal volume from distinct plots do not account for the same number of observations. With the same aim of facilitating the
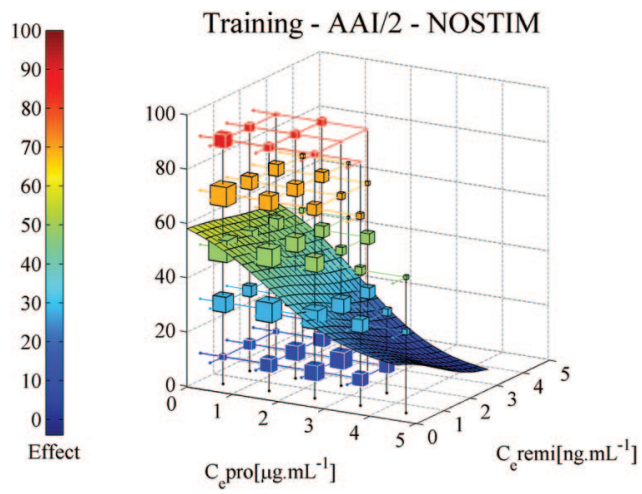

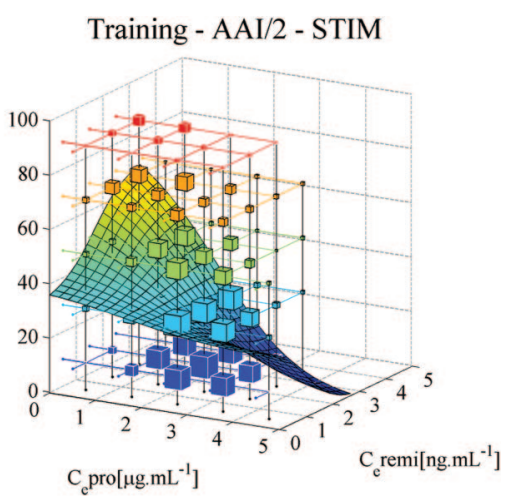

Figure 1. Model of the relationship between effect-site propofol and effect-site remifentanil with respect to the autoregressive auditory evoked potential index. The graph on the left shows the relation when there is no noxious stimulation to the patient, and the graph on the right when there is noxious stimulation. Observations are grouped in cubes whose sizes are proportional to the number of observations. The colored surface corresponds to the predictions of the model and the intensity of the color relates to the level of effect. Note that the origin of each graph, the predictions when there is no drug given, is on the lower left corner. 

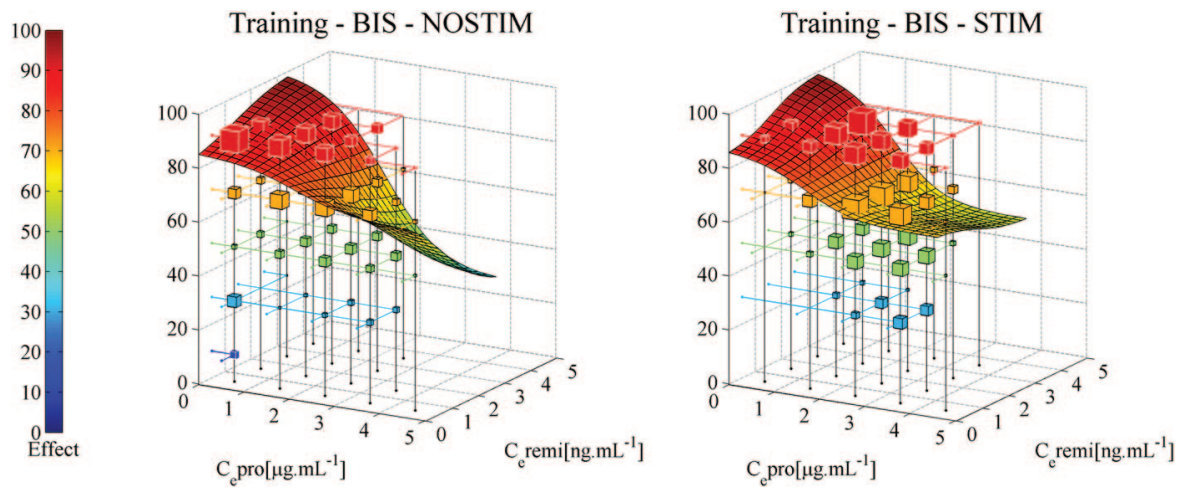

Figure 2. Model of the relationship between effect-site propofol and effect-site remifentanil with respect to bispectral index. The graph on the left shows the relation when there is no noxious stimulation to the patient, and the graph on the right when there is noxious stimulation. Observations are grouped in cubes whose sizes are proportional to the number of observations. The colored surface corresponds to the predictions of the model and the intensity of the color relates to the level of effect. Note that the origin of each graph, the predictions when there is no drug given, is on the lower left corner.
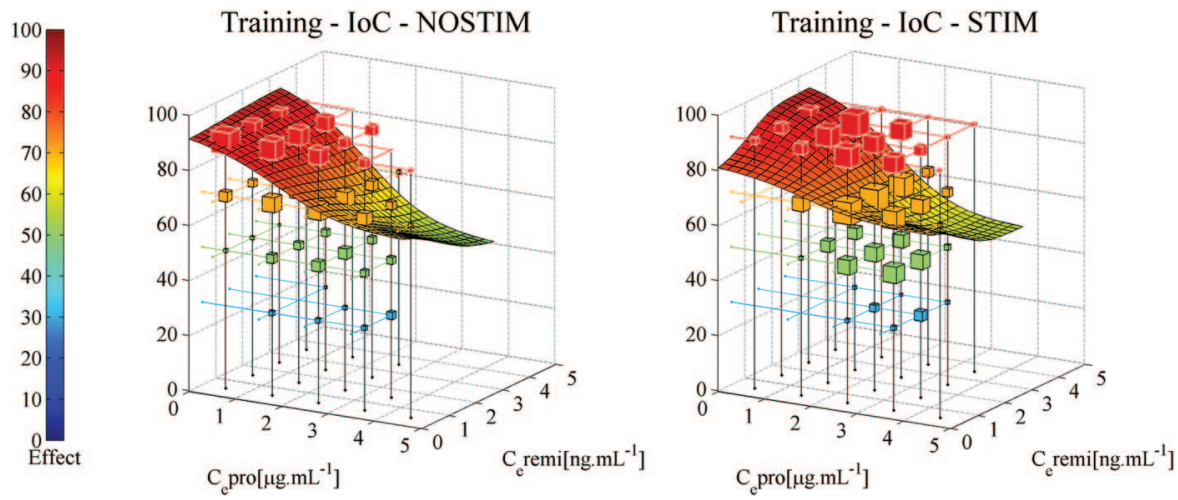

Figure 3. Model of the relationship between effect-site propofol and effect-site remifentanil with respect to index of consciousness. The graph on the left shows the relation when there is no noxious stimulation to the patient, and the graph on the right when there is noxious stimulation. Observations are grouped in cubes whose sizes are proportional to the number of observations. The colored surface corresponds to the predictions of the model and the intensity of the color relates to the level of effect. Note that the origin of each graph, the predictions when there is no drug given, is on the lower left corner.

\begin{tabular}{|c|c|c|c|c|c|c|}
\hline & & NOSTIM & & & STIM & \\
\hline & AAI $/ 2$ & BIS & IoC & $\mathrm{AAI} / 2$ & BIS & loc \\
\hline \multicolumn{7}{|l|}{$\begin{array}{l}\text { Training } \\
\text { group }\end{array}$} \\
\hline MDAPE & 32.87 & 12.89 & 8.77 & 39.57 & 12.88 & 12.43 \\
\hline MDRMSE & 17.01 & 12.18 & 9.40 & 15.31 & 11.19 & 10.34 \\
\hline MDPE & -1.86 & 3.97 & 2.21 & -17.05 & 2.86 & 0.49 \\
\hline \multicolumn{7}{|l|}{$\begin{array}{l}\text { Validation } \\
\text { group }\end{array}$} \\
\hline MDAPE & 34.81 & 14.78 & 10.25 & 33.71 & 15.85 & 12.85 \\
\hline MDRMSE & 16.81 & 15.91 & 11.81 & 12.39 & 13.25 & 11.50 \\
\hline MDPE & -8.37 & 5.65 & -1.43 & -18.69 & -5.83 & -8.16 \\
\hline
\end{tabular}

NOSTIM = absence of noxious stimulation; STIM = presence of noxious stimulation; $\mathrm{AAI} / 2$ = autoregressive auditory evoked potential index; $\mathrm{BIS}=$ bispectral index; IoC = index of consciousness; PE = (Obs - Pred)/Pred: percentage performance error; MDAPE = median of individual median absolute $\mathrm{PE}$, indicator of inaccuracy; MDRMSE = median of individual root mean squared error, indicator of inaccuracy; MDPE = median of individual median $\mathrm{PE}$, indicator of bias.

interpretation of the graphs, each cube is colored according to the measured effect and orthogonal projections are drawn.

The prediction of the ANFIS model is represented by the colored surface. In all figures, the graph on the left side shows the model estimated for the data collected without noxious stimulation whereas the graph to the right shows the model estimated when there was noxious stimulation. Figure 1 shows the results for AAI/2, Figure 2 for BIS, and Figure 3 for IoC. For each measure of effect, 2 models were constructed depending on the presence or absence of noxious stimulation.

Table 3 summarizes the values of MDAPE, MDRMSE, and MDPE for every EEG-derived measure of effect studied under stimulation and without noxious stimulation. For the training group, $\mathrm{AAI} / 2$ presents median values of the individual MDAPE $>30 \%$ whereas BIS and IoC values are approximately $10 \%$. Similar values of MDRMSE are found for the 3 indexes. Regarding MDPE for the training group, the median of the individual MDPE is globally well centered, suggesting very little bias in the individuals of the training group, except for $\mathrm{AAI} / 2$ under stimulation.

Table 4 shows the $P_{k}$ values calculated for every effect measure, including $\mathrm{C}_{\mathrm{e}}$ pro, $\mathrm{C}_{\mathrm{e}}$ remi, as well as $\mathrm{HR}$ and systolic ABP, to predict the RSS score.

\section{Prospective Validation Group}

A total of 68 patients were included in the prospective validation group. Demographics of these patients are summarized in Table 2. The predicted effect-site concentrations 
Table 4. $P_{k}$ Comparison: Ability of Each Measure of Effect to Predict the Observed Ramsay Sedation Scale Score

\begin{tabular}{lccccccc} 
& AAI/2 & BIS & loC & C e pro & C $_{\mathrm{e}}$ remi & HR & ABP \\
$\begin{array}{l}\text { Training group } \\
\quad P_{k}(\mathrm{SE})\end{array}$ & $0.82(0.01)$ & $0.81(0.01)$ & $0.85(0.01)$ & $0.75(0.01)$ & $0.59(0.02)$ & $0.63(0.01)$ & $0.69(0.02)$ \\
$\begin{array}{l}\text { Validation group } \\
\quad P_{k}(\mathrm{SE})\end{array}$ & $0.81(0.01)$ & $0.80(0.01)$ & $0.80(0.01)$ & $0.66(0.01)$ & $0.67(0.01)$ & $0.6(0.02)$ & $0.64(0.02)$ \\
\hline
\end{tabular}

Data from STIM (presence of noxious stimulation) and NOSTIM (absence of noxious stimulation) are pooled.

$\mathrm{SE}=$ standard error of $\mathrm{P}_{\mathrm{k}} ; \mathrm{AAI} / 2=$ autoregressive auditory evoked potential index; $\mathrm{BIS}=$ bispectral index; loC = index of consciousness; $\mathrm{C}_{\mathrm{e}}$ pro = effect-site propofol; $\mathrm{C}_{\mathrm{e}}$ remi $=$ effect-site remifentanil; $\mathrm{HR}=$ heart rate; $\mathrm{ABP}=$ arterial blood pressure.

achieved in this group of patients ranged from 0 to 3.5 $\mu \mathrm{g} \cdot \mathrm{mL}^{-1}$ for propofol and 0 to $2 \mathrm{ng} \cdot \mathrm{mL}^{-1}$ for remifentanil. The median (range) duration of the procedure was 63 minutes (24-124 minutes).

Eight hundred twenty evaluations of RSS score were performed in the 68 patients from the prospective validation group. The median number of evaluations per patient was 12. From those 820, 483 were performed with the patient not being stimulated whereas the remaining 337 were performed while the patient had the endoscopy tube inside and the exploration was being performed.

With respect to neurophysiological measures, approximately 25,000 data points were collected (approximately 10,000 without stimulation and 15,000 with stimulation).

Table 3 shows the overall values of MDAPE, MDRMSE, and MDPE allowing a comparison between training and validation. As can be seen in the table, the values of MDAPE and MDRMSE are similar to those calculated in the training group, suggesting a similar degree of accuracy of the model to predict the observations from a different set of patients. The distribution of the individual values of MDPE suggests an increase in the bias of the model to prospectively predict the new individuals of the prospective validation group.

Table 4 shows the $P_{k}$ values calculated for every effect measure to predict the RSS score in the validation group. $\mathrm{P}_{\mathrm{k}}$ values were calculated pooling the STIM and NO STIM data. The values reported suggest good prediction ability

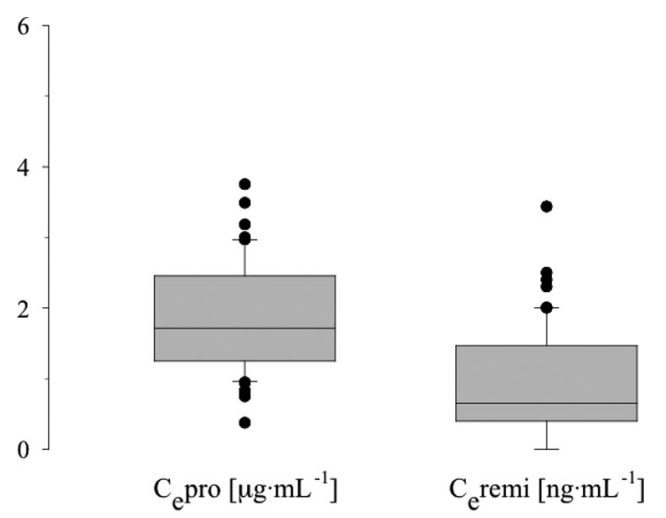

Figure 4. Observed effect-site propofol ( $\mathrm{C}_{\mathrm{e}}$ pro) and effect-site remifentanil ( $\mathrm{C}_{\mathrm{e}}$ remi) associated to a Ramsay Sedation Scale score of 4 in the training group. The thick line represents the median value, the box contains the range of predicted concentrations between the 25th and 75th percentiles, and the whiskers the 5th and 95th percentiles. This range of concentrations will be fed to the model to obtain predictions of the expected effect on autoregressive auditory evoked potential index, bispectral index, and index of consciousness. for $\mathrm{AAI} / 2, \mathrm{BIS}$, and IoC in the prospective validation group patients.

\section{Model Application}

Figure 4 shows the distribution of predicted $\mathrm{C}_{\mathrm{e}}$ pro and $\mathrm{C}_{\mathrm{e}}$ remi that had an associated measured RSS score of 4 in the patients of the training group. Those median values of $\mathrm{C}_{\mathrm{e}}$ pro and $\mathrm{C}_{\mathrm{e}}$ remi were used as input to the model to estimate the predictions of $\mathrm{AAI} / 2$, BIS, and IoC associated with an RSS score of 4; the results are shown in Figure 5. Noxious stimulation implies an increase in the target $C_{\mathrm{e}}$ pro and $\mathrm{C}_{\mathrm{e}}$ remi to achieve the same level of sedation.

\section{DISCUSSION}

This study demonstrates that to achieve and maintain an RSS score of 4 , remifentanil administration reduces the required propofol predicted concentrations. The models predict that for those concentrations, the values of BIS, $\mathrm{AAI} / 2$, and $\mathrm{IoC}$ are 71 to 75,25 to 30 , and 72 to 76 , respectively. At the concentrations of propofol and remifentanil used in the study, it has been possible to evaluate almost all possible combinations of both drugs and their corresponding sedative effects. The presence of stimulation required an increase in drug concentration targets to maintain the same level of effect. IoC, BIS, and AAI/2 exhibited good performance to control sedation based on their $P_{k}$ values.

The values for MDAPE, MDRMSE, and MDPE in the training group show that the model selected is able to effectively describe the data recorded without stimulation, based on good accuracy and almost no bias. The model is robust in that approximately the same values for MDAPE, MDRMSE, and MDPE are calculated when it predicts the observations in the prospective validation group, as can be seen in Table 3. The AAI/2 presents higher MDAPE values than BIS and IoC. The similar values of MDRMSE presented by the 3 indexes show that this larger MDAPE for $\mathrm{AAI} / 2$ is likely attributable to the fact that a given level of sedation produces higher values for BIS and IoC as compared with $\mathrm{AAI} / 2$. For the same reason, $\mathrm{AAI} / 2$ tends to show higher MDPE values. The model for AAI/2, BIS, and IoC defined under stimulation shows similar accuracy and bias.

The behavior of the monitors of the level of sedation can be assessed by observing their ability to predict the RSS score as quantified by the $P_{k}$ statistic. All 3 exhibited a good predictive ability, both in the training and in the prospective validation groups. The training group IoC had a $P_{k}$ value of 0.85 (AAI/2: 0.82 and BIS: 0.81 ), whereas in the 
Figure 5. Model effect predictions for the autoregressive auditory evoked potential index (AAI/2), bispectral index (BIS), and index of consciousness (loC) effect induced by the median effect-site propofol ( $\mathrm{C}_{\mathrm{e}}$ pro) and effect-site remifentanil $\left(\mathrm{C}_{\mathrm{e}}\right.$ remi) associated with a Ramsay Sedation Scale score of 4 . Each graph shows the plots of the isobologram for the same level of hypnotic effect in the absence (continuous line, NOSTIM) and presence (dashed line, STIM) of noxious stimulation.
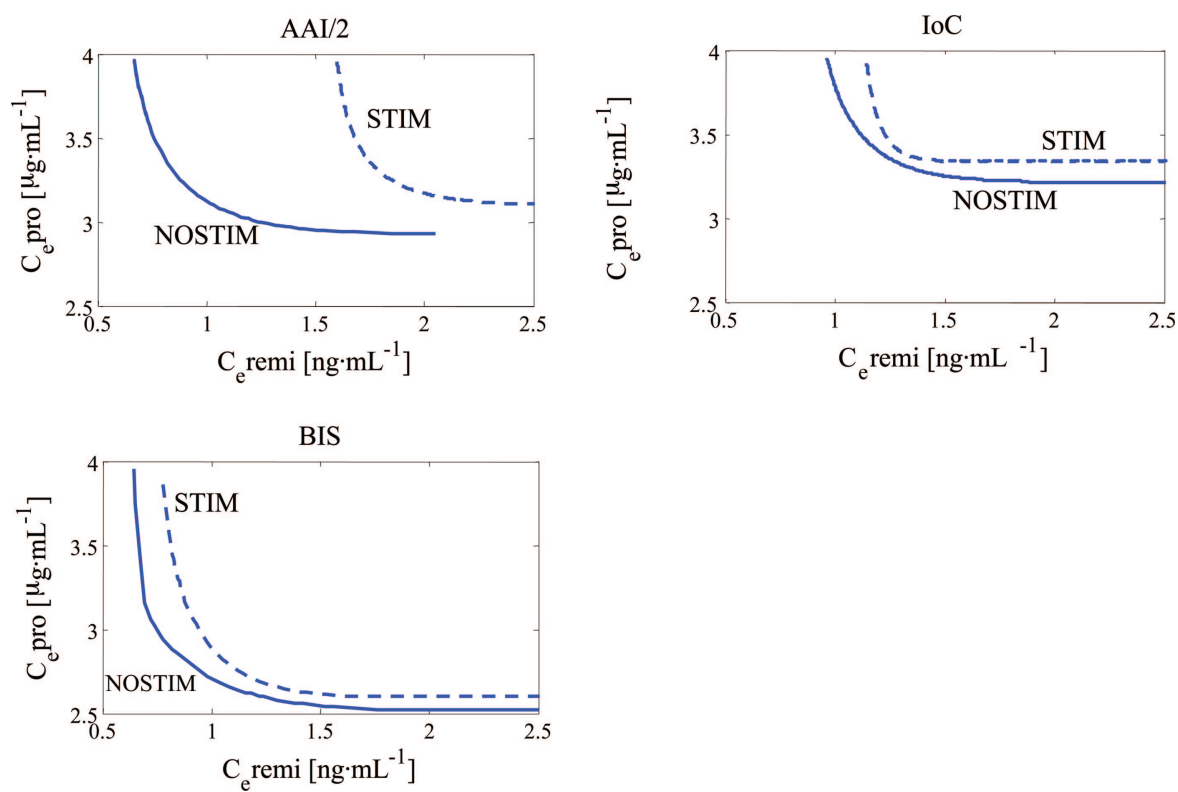

prospective validation, $\mathrm{AAI} / 2$ had a $\mathrm{P}_{\mathrm{k}}$ of 0.81 (BIS: 0.80 and IoC: 0.80$)$. Such values of $P_{k}$ are slightly lower than those reported by other authors, which could be explained by the fact that our study was performed in real patients with real noxious stimuli applied under clinical conditions, which is not as well controlled as a volunteer study. Also, the present study was performed under non-steady-state conditions. Hemodynamic parameters had $\mathrm{P}_{\mathrm{k}}$ values $<0.7$ in both groups and $\mathrm{C}_{\mathrm{e}}$ pro 0.75 in the training group and 0.66 in the prospective validation group.

Struys et al. ${ }^{18}$ studied the ability of BIS and AAI to predict sedative responses (OAA/S scale, AAI, and BIS) induced by a gradually increasing TCI of propofol targeting the biophase, starting at $1 \mu \mathrm{g} \cdot \mathrm{mL}^{-1}$ and up to $8 \mu \mathrm{g} \cdot \mathrm{mL}^{-1}$ under 3 different concentrations of remifentanil $\left(0,2\right.$, and $\left.4 \mathrm{ng} \cdot \mathrm{mL}^{-1}\right)$, a different design from the "crisscross" used in the present work. The ability of AAI and BIS to predict OAA/S scores as quantified by their pooled $P_{k}$ values were 0.87 and 0.9 , significantly higher values than in our study.

Using $\mathrm{C}_{\mathrm{e}}$ pro and $\mathrm{C}_{\mathrm{e}}$ remi as input to the model, the expected values for $\mathrm{AAI} / 2$, BIS, and IoC corresponding to RSS score 4 can be estimated and used as objective targets to predict the desired sedation level without applying any stimulation to the patient to assess the level of sedation.

The effect of stressful stimulation as in this case, the introduction of the endoscopy probe through the mouth, pharynx, and upper digestive tract of the patient, shifts the shape of the surface upward of all sedative effects studied, suggesting that increased drug requirements are necessary to maintain the same level of sedation than without stimulation. It can be better assessed by looking at Figure 5 where for $\mathrm{AAI} / 2$, BIS, and IoC, an influence of noxious stimulation can be seen. This effect is more evident in the case of $\mathrm{AAI} / 2$, where a value of 25 to 30 can be achieved with $\mathrm{C}_{\mathrm{e}}$ pro 3.8 to $2.9 \mu \mathrm{g} \cdot \mathrm{mL}^{-1}$ whereas $\mathrm{C}_{\mathrm{e}}$ remi increases from 0.7 to $2 \mathrm{ng} \cdot \mathrm{mL}^{-1}$. When there is noxious stimulation, $\mathrm{C}_{\mathrm{e}}$ pro shifts from 4 to $3.2 \mu \mathrm{g} \cdot \mathrm{mL}^{-1}$, whereas $\mathrm{C}_{\mathrm{e}}$ remi increases from 1.6 to $2.5 \mathrm{ng} \cdot \mathrm{mL}^{-1}$. Although present, differences are not so obvious for BIS and IoC.
Nieuwenhuijs et al. ${ }^{19}$ have studied the interaction between propofol and remifentanil with respect to BIS. They concluded that for propofol concentrations up to $2 \mu \mathrm{g} \mathrm{mL}-1$ combined with remifentanil concentrations ranging from 0 to $2 \mathrm{ng} \mathrm{mL} \mathrm{m}^{-1}$, both lower than in our study, there is no synergy with respect to BIS as a measure of effect. In the present work, no formal estimation of synergy was attempted, although the bowing shape of the surfaces relating concentrations and the different effects suggest that there might be a positive interaction. Bouillon et al. ${ }^{20}$ also studied the interaction between propofol and remifentanil with respect to hypnotic responses such as absence of response to shaking and shouting, BIS, and approximate entropy of the EEG. They concluded that the addition of remifentanil decreases the concentrations of propofol required, whereas remifentanil alone, only if given at very high concentrations and beyond those used clinically, might induce any sign of sedation. Our study differs in that our patients underwent real exploration with painful stimuli that could be intense at some points, unlike a well-controlled volunteer study.

Kazama et al. ${ }^{21}$ estimated a propofol $50 \%$ concentration value for loss of consciousness in patients undergoing gastroscopy ranging from 2.23 to $1.40 \mu \mathrm{g} \mathrm{mL} \mathrm{m}^{-1}$ depending on the age of the patients; the younger, the more propofol required. These values are consistent with our estimation for RSS score 4 , which associates unconsciousness to tolerate insertion of the gastroscope.

A close observation of model graphs shows that when remifentanil is given alone or administered with concentrations of propofol $<1.4 \mu \mathrm{g} \mathrm{mL}^{-1}$, there is a tendency to predict an increase of the values of the indicators calculated from the EEG effects. A plausible explanation could be the presence of electromyographic artifact caused by a relatively small contribution of the sedative component of propofol during introduction of the tube. Another explanation is based on the muscular rigidity associated with the use of powerful opioids such as remifentanil. Although rigidity was not clinically observed in any of the patients studied, remifentanil might increase muscular tone to the point that it might alter the EEG 
waves. It must be kept in mind that logically no neuromuscular blocking drug was used because it was mandatory that all patients breathe by themselves. In most studies using the EEG as a surrogate measure of opioid drug effect, the patients or volunteers were administered low concentrations of neuromuscular blocking drug to avoid rigidity and artifacts on the EEG recording.

This work uses ANFIS, a fuzzy reasoning-based method of data analysis. Other authors have used a similar approach based on fuzzy logic principles to analyze complex data from clinical studies. ${ }^{22,23}$ The ANFIS approach allows the implementation of very complex processes, where a simple mathematical model cannot be obtained. Fuzzy logic has been successfully applied to highly nonlinear systems, where it is observed to greatly simplify the modeling process. The power of fuzzy logic is to perform reasonable and meaningful operations on concepts that cannot be easily codified using a classical mathematical approach. Such modification allows for a much more flexible and widespread use of reliable and consistent logic in a variety of applications. The most common use of fuzzy logic lies in the field of control systems, although the theory seems to have serious potential in the different fields of artificial intelligence. It can be used as a preliminary approach before conducting a more sophisticated pharmacologic analysis such as a population approach using mixed effects modeling techniques. Zhang and Roy ${ }^{14}$ have used an ANFIS approach to extract from the EEG parameters that allow discrimination between awake and asleep states in anesthetized dogs. To make our results more solid, we have attempted to prospectively validate the ANFIS model in a new set of data, obtaining similar results. This means that the model could be used safely to predict the effect of propofol and remifentanil in this kind of procedure and patient.

To conclude, we have used an ANFIS modeling approach with prospective validation to study the relationship between propofol and remifentanil predicted concentrations and sedative effects in patients undergoing USE. Optimal sedation can be achieved with a target propofol biophase concentration of 2.8 to $1.8 \mu \mathrm{g} \mathrm{mL} \mathrm{mL}^{-1}$ with remifentanil increasing from 0 to $1.5 \mathrm{ng} \mathrm{mL}^{-1}$. The IoC value associated with such concentrations is 72 to 76 , similar to BIS 71 to 75 , whereas the interval for $\mathrm{AAI} / 2$ is 25 to 30 . The presence of stimulation requires an increase in the target concentration of propofol and remifentanil to maintain patients at the same level of sedation. $H$

\section{DISCLOSURES}

Name: P.L. Gambús, MD.

Contribution: Principal investigator, study design, data collection, data analysis, and preparation of the manuscript.

Conflict of Interest: This author reported no conflicts of interest.

Name: E.W. Jensen, MSc, PhD.

Contribution: Study design, data analysis, and preparation of the manuscript.

Conflict of Interest: This author is an employee of Aircraft Medical, the owners of IoC.

Name: M. Jospin, MSc.

Contribution: Data analysis and preparation of the manuscript. Conflict of Interest: This author is an employee of Aircraft Medical, the owners of IoC.
Name: X. Borrat, MD.

Contribution: Data collection and preparation of the manuscript. Conflict of Interest: This author reported no conflicts of interest.

Name: G. Martínez Pallí, MD.

Contribution: Data collection

Conflict of Interest: This author reported no conflicts of interest.

Name: J. Fernández-Candil, MD.

Contribution: Study design and preparation of the manuscript.

Conflict of Interest: This author reported no conflicts of interest.

Name: J.F. Valencia, MSc.

Contribution: Data analysis and preparation of the manuscript. Conflict of Interest: This author reported no conflicts of interest.

Name: X. Barba, CRNA.

Contribution: Data collection.

Conflict of Interest: This author reported no conflicts of interest.

Name: P. Caminal, MSc, PhD.

Contribution: Study design.

Conflict of Interest: This author reported no conflicts of interest.

Name: I.F. Trocóniz, PhD.

Contribution: Data analysis and preparation of the manuscript.

Conflict of Interest: This author reported no conflicts of interest.

\section{ACKNOWLEDGMENTS}

The help of Stephane Ruton from FreseniusVial, Chemin de Fer, Béziers, France, who generously lent us the TCI system (Base Primea) used in this project, is greatly appreciated.

\section{REFERENCES}

1. Shafer SL, Gregg KM. Algorithms to rapidly achieve and maintain stable drug concentrations at the site of drug effect with a computer-controlled infusion pump. J Pharmacokinet Biopharm 1992;20:147-69

2. Ramsay MAE, Savege TM, Simpson BRJ, Goodwin R. Controlled sedation with alphaxalone-alphadolone. BMJ 1974;2: $656-9$

3. Chernik DA, Gillings D, Laine H, Hendler J, Silver JM, Davidson AB, Schwam EM, Siegel JL. Validity and reliability of the Observer's Assessment of Alertness/Sedation Scale: study with intravenous midazolam. J Clin Psychopharmacol 1990;10: 244-51

4. Billard V, Gambús PL, Chamoun N, Stanski DR, Shafer SL. A comparison of spectral edge, delta power, and bispectral index as EEG measures of alfentanil, propofol, and midazolam drug effect. Clin Pharmacol Ther 1997;61:45-58

5. Jensen EW, Lindholm P, Henneberg SW. Autoregressive modeling with exogenous input of middle-latency auditory-evoked potentials to measure rapid changes in depth of anesthesia. Methods Inf Med 1996:35:256-60

6. Takagi T, Sugeno M. Fuzzy identification of systems and its applications to modelling and control. IEEE Trans Syst Man Cybern 1985;15:116-32

7. Jang JSR. ANFIS: adaptive network-based fuzzy inference system. IEEE Trans Syst Man Cybern 1993;23:665-85

8. Short TG, Ho TY, Minto CF, Schnider TW, Shafer SL. Efficient trial design for eliciting a pharmacokinetic-pharmacodynamic model-based response surface describing the interaction between two intravenous anesthetic drugs. Anesthesiology 2002;96: $400-8$

9. Schnider TW, Minto CF, Shafer SL, Gambús PL, Andresen C, Goodale DB, Youngs EJ. The influence of age on propofol pharmacodynamics. Anesthesiology 1999;90:1502-16 
10. Schnider TW, Minto CF, Gambús PL, Andresen C, Goodale DB, Shafer SL, Youngs EJ. The influence of method of administration and covariates on the pharmacokinetics of propofol in adult volunteers. Anesthesiology 1998;88: $1170-82$

11. Minto CF, Schnider TW, Egan TD, Youngs E, Lemmens HJ, Gambús PL, Billard V, Hoke JF, Moore KH, Hermann DJ, Muir KT, Mandema JW, Shafer SL. Influence of age and gender on the pharmacokinetics and pharmacodynamics of remifentanil. I. Model development. Anesthesiology 1997;86:10-23

12. Revuelta M, Paniagua P, Campos JM, Fernandez JA, Martinez A, Jospin M, Litvan $\mathrm{H}$. Validation of the index of consciousness during sevoflurane and remifentanil anaesthesia: a comparison with the bispectral index and the cerebral state index. Br J Anaesth 2008;101:653-8

13. Jensen EW, Jospin M, Gambús PL, Vallverdu M, Caminal P. Validation of the Index of Consciousness (IoC) during sedation/analgesia for ultrasonographic endoscopy. Conf Proc IEEE Eng Med Biol Soc 2008;2008:5552-5

14. Zhang XS, Roy RJ. Derived fuzzy knowledge model for estimating the depth of anesthesia. IEEE Trans Biomed Eng 2001;48:312-23

15. Varvel JR, Donoho DL, Shafer SL. Measuring the predictive performance of computer-controlled infusion pumps. J Pharmacokinet Biopharm 1992;20:63-94

16. Smith WD, Dutton RC, Smith NT. Measuring the performance of anesthetic depth indicators. Anesthesiology 1996; 84:38-51

17. Ellerkmann RK, Bruhn J, Soehle M, Kehrer M, Hoeft A, Kreuer S. Maximizing prediction probability $\mathrm{PK}$ as an alternative semiparametric approach to estimate the plasma effect-site equilibration rate constant ke0. Anesth Analg 2009;109:1470-8
18. Struys MM, Vereecke H, Moerman A, Jensen EW, Verhaeghen D, De Neve N, Dumortier FJ, Mortier EP. Ability of the bispectral index, autoregressive modelling with exogenous input-derived auditory evoked potentials, and predicted propofol concentrations to measure patient responsiveness during anesthesia with propofol and remifentanil. Anesthesiology 2003;99:802-12

19. Nieuwenhuijs DJ, Olofsen E, Romberg RR, Sarton E, Ward D, Englbers F, Vuyk J, Mooren R, Teppema LJ, Dahan A. Response surface modeling of remifentanil-propofol interaction on cardiorespiratory control and bispectral index. Anesthesiology 2003;98:312-22

20. Bouillon TW, Bruhn J, Radulescu L, Andresen C, Shafer TJ, Cohane C, Shafer SL. Pharmacodynamic interaction between propofol and remifentanil regarding hypnosis, tolerance of laryngoscopy, bispectral index, and electroencephalographic approximate entropy. Anesthesiology 2004;100:1353-72

21. Kazama T, Takeuchi K, Ikeda K, Ikeda T, Kikura M, Iida T, Suzuki S, Hanai H, Sato S. Optimal propofol plasma concentration during upper gastrointestinal endoscopy in young, middleaged, and elderly patients. Anesthesiology 2000;93: 662-9

22. Kern SE, Johnson JO, Westenskow DR. Fuzzy logic for model adaptation of a pharmacokinetic-based closed loop delivery system for pancuronium. Artif Intell Med 1997;11:9-31

23. Kang SH, Poynton MR, Kim KM, Lee H, Kim DH, Lee SH, Bae KS, Linares O, Kern SE, Noh GJ. Population pharmacokinetic and pharmacodynamic models of remifentanil in healthy volunteers using artificial neural network analysis. Br J Clin Pharmacol 2007;64:3-13 